\title{
Comparison of safety and outcomes of shock wave lithotripsy between elderly and non-elderly patients
}

\author{
Yi-Zhong Chen' \\ Wun-Rong Lin ${ }^{1,2}$ \\ Chih-Chiao Lee ${ }^{1,2}$ \\ Fang-Ju Sun ${ }^{1,3}$ \\ Yung-Chiong Chow ${ }^{1,2}$ \\ Wei-Kung Tsai ${ }^{1,2}$ \\ Pai-Kai Chiang ${ }^{1,2}$ \\ Ting-Po Lin ${ }^{1,2}$ \\ Marcelo Chen ${ }^{1-3}$ \\ Allen W Chiu ${ }^{1,4}$ \\ 'Department of Urology, MacKay \\ Memorial Hospital, ${ }^{2} \mathrm{School}$ \\ of Medicine, MacKay Medical \\ College, ${ }^{3}$ Department of Cosmetic \\ Applications and Management, \\ Mackay Junior College of Medicine, \\ Nursing and Management, ${ }^{4}$ School \\ of Medicine, National Yang-Ming \\ University, Taipei, Taiwan
}

This article was published in the following Dove Press journal:

Clinical Interventions in Aging

II April 2017

Number of times this article has been viewed

Background: This study compared the clinical outcomes of extracorporeal shock wave lithotripsy between elderly (aged $\geq 65$ years) and non-elderly (aged $<65$ years) patients.

Methods: A retrospective review of medical records was performed on 483 (non-elderly: 245, elderly: 238) patients with upper urinary tract stones who underwent shock wave lithotripsy between 2007 and 2015. The demographic data, stone parameters, stone-free rate, retreatment rate, and complication rate were analyzed in both elderly and non-elderly patient groups.

Results: There was no significant difference between non-elderly and elderly patients in terms of stone-free rate $(46.5 \%$ vs $41.1 \%, P>0.05)$ regardless of stone site or stone size and overall retreatment rate $(41.6 \%$ vs $37.0 \%, P>0.05)$. Elderly patients had a higher complication rate than non-elderly patients $(15.5 \%$ vs $23.5 \%, P=0.026)$. The most common complication was flank pain. Receiver operating characteristic curves predicted that elderly patients (cutoff value: 65 years of age) had a higher risk of complications and that patients with smaller stones (cutoff value: $0.8 \mathrm{~cm}$ ) had a higher stone-free rate.

Conclusion: This study showed that elderly patients with upper urinary tract stones undergoing shock wave lithotripsy had comparable efficacy for stone-free rates and retreatment rates, but higher complication rates.

Keywords: elderly, shock wave lithotripsy, upper urinary tract stone, safety, outcome

\section{Introduction}

Extracorporeal shock wave lithotripsy (ESWL) has revolutionized the treatment of noninfectious ureter and renal stones since the 1980s, and it is the least invasive treatment and most common intervention for upper urinary tract stones. ${ }^{1}$ Advances in shock wave lithotripsy (SWL) technology have improved treatment outcomes and reduced complications, which have led to its widespread use. The world's elderly population is growing at a rapid rate. In 2015, people aged $\geq 65$ years accounted for $8.5 \%$ of the world's population, and this proportion is expected to rise to $17 \%$ by the year $2050 .^{2}$ With the growing number of elderly patients and their increased life expectancy, medical practitioners must determine whether treatment modalities used in the general population are also applicable to the elderly. The prevalence and incidence of renal stones have been reported to be increasing across the world. ${ }^{3}$ Inevitably, the number of elderly patients seeking medical help for urolithiasis will continue to rise. Medical practitioners will consider SWL as the primary treatment option in many of these patients; however, data on SWL efficacy and safety in elderly patients are scarce. The aim of this study was to compare the efficacy and safety of SWL between the elderly and non-elderly.
Correspondence: Marcelo Chen Department of Urology, MacKay Memorial Hospital, No 92, Sec 2, Zhongshan N Road, Zhongshan District, Taipei 104, Taiwan Tel +886 225433535 Ext 9 Fax +886 225433535 Ext 2561 Email mchen4270@yahoo.com 


\section{Patients and methods}

This study retrospectively reviewed the medical records of 483 patients with upper urinary tract stones who underwent SWL between March 2007 and December 2015. The number of elderly patients (aged $\geq 65$ years) was 238 , and the number of non-elderly patients (aged $<65$ years) was 245 . Although there were more elderly patients with upper urinary tract stones who needed SWL during the study period, some patients refused SWL because of worries about possible higher risks associated with old age. Therefore, only 238 elderly patients underwent SWL, and they were all included in this study. For comparative purposes, a similar number of non-elderly patients who underwent SWL were randomly selected. In patients who underwent more than one session of SWL during the study period, only the first SWL session was investigated in this study. Patients underwent SWL with either an electrohydraulic lithotripter (E3000; Medispec LTD, Yehud, Israel) or an electromagnetic lithotripter (EM1000; Medispec LTD) at two branches of the hospital depending on their location. However, all procedures were performed by the same two technicians who had experience in operating both the machines. The exclusion criteria were patients with congenital anomalies, urinary diversion, urosepsis, or coagulopathy or those who had undergone previous interventions for the urinary tract stones. The energy level was gradually increased from 14 to $22 \mathrm{KV}$, and shock waves were delivered at a rate of 120 shocks per minute by both the lithotripters. The number of shock waves in one session ranged from 3,000 to 4,000 , depending on stone fragmentation and patient tolerance. Patients who received SWL by electrohydraulic lithotripters were placed under consciously monitored intravenous sedation with $100-150 \mathrm{mg}$ of fentanyl and $2.5-3 \mathrm{~mL}$ of midazolam depending on body weight, whereas those treated with electromagnetic lithotripters were treated without anesthesia.

All the patients underwent physiological and laboratory examinations before ESWL. Stone size was measured on plain abdominal radiograph of kidney, ureter, and bladder (KUB) in one dimension. Patients were evaluated within 4 weeks after SWL by KUB. Stone-free status was defined as the absence of stone on radiographic imaging, and retreatment was defined as further surgical intervention for residual stone fragments $>0.5 \mathrm{~cm}$. Complications were classified according to the Clavien-Dindo classification. All data were compared using independent two-sample $t$-test and chi-square test. Receiver operating characteristic (ROC) curves were generated for stone-free rates and complications of upper urinary tract stone cases separately, and cutoff points were determined with respect to the greatest sensitivity and specificity values. Statistical significance was set at $P<0.05$.

The use of data and the research protocol of the study were permitted and approved by the Mackay Memorial Hospital Institutional Review Board. Patients' consent to review their medical records was not required by Mackay Memorial Hospital Institutional Review Board. According to the regulations of Mackay Memorial Hospital Institutional Review Board, collection of noninvasive materials for routine practice and data prepared retrospectively for future publications does not require informed consent. All personal information was de-identified prior to data analysis, thus covering patient data confidentiality.

\section{Results}

The mean age was $48.2 \pm 9.7$ years in the non-elderly group and $72.9 \pm 6.0$ years in the elderly group $(P<0.05)$. The elderly group had a higher proportion of kidney stones $(P<0.05)$. Comparison between the two groups showed significantly larger stones in the elderly group (kidney, $0.94 \pm 0.31 \mathrm{~cm}$ in non-elderly group and $1.26 \pm 0.69 \mathrm{~cm}$ in elderly group, $P<0.05$; ureter, $0.83 \pm 0.28 \mathrm{~cm}$ in non-elderly group and $0.92 \pm 0.47 \mathrm{~cm}$ in elderly group, $P<0.05$ ). The electrohydraulic - electromagnetic ratio between the two groups was not significantly different $(P>0.05)$ (Table 1). Two elderly patients did not have a follow-up on KUB and did not receive further treatment. In the subgroup analysis, there was no significant difference in the stone-free rates, retreatment rates, and complication rates between patients undergoing SWL with the electrohydraulic and electromagnetic lithotripters.

The overall stone-free rates were similar (non-elderly $46.5 \%$ vs elderly $41.1 \%, P=0.23$ ). Comparable stone-free rates were seen for renal stones (non-elderly $40.3 \%$ vs

Table I Patient demographics and stone characteristics

\begin{tabular}{|c|c|c|c|}
\hline Variables & $\begin{array}{l}\text { Patients aged } \\
<65 \text { years }\end{array}$ & $\begin{array}{l}\text { Patients aged } \\
\geq 65 \text { years }\end{array}$ & $P$-value \\
\hline Number of patients & 245 & 238 & 0.29 \\
\hline $\begin{array}{l}\text { Patient age, years } \\
\text { (mean } \pm \text { SD) }\end{array}$ & $48.2 \pm 9.7$ & $72.9 \pm 6.0$ & $<0.001$ \\
\hline Stone site & & & 0.006 \\
\hline Kidney, n (\%) & $119(48.6)$ & $145(60.9)$ & \\
\hline Ureter, n (\%) & $126(5 \mid .4)$ & $93(39.1)$ & \\
\hline Stone size, $\mathrm{cm}($ mean $\pm \mathrm{SD})$ & $0.89 \pm 0.30$ & $1.13 \pm 0.63$ & $<0.001$ \\
\hline SWL & & & 0.337 \\
\hline Electrohydraulic (n) & 163 & 168 & \\
\hline Electromagnetic (n) & 82 & 70 & \\
\hline
\end{tabular}

Abbreviations: SD, standard deviation; SWL, shock wave lithotripsy. 
Table 2 Comparison of stone-free rates by stone site and size

\begin{tabular}{|c|c|c|c|}
\hline Variables & $\begin{array}{l}\text { Patients aged } \\
<65 \text { years, } n(\%)\end{array}$ & $\begin{array}{l}\text { Patients aged } \\
\geq 65 \text { years, } \mathrm{n}(\%)\end{array}$ & $P$-value \\
\hline Overall & I |4/245 (46.5) & $97 / 236^{a}(4 I .1)$ & 0.230 \\
\hline Kidney & $48 / 119(40.3)$ & $48 / 143(33.5)$ & 0.257 \\
\hline Ureter & $66 / 126(52.3)$ & $49 / 93(52.7)$ & 0.964 \\
\hline Stone size $<8 \mathrm{~mm}$ & $53 / 92(57.6)$ & $46 / 75$ (6I.3) & 0.626 \\
\hline Kidney & I6/33 (48.4) & I7/30 (56.7) & 0.516 \\
\hline Ureter & $37 / 59(62.7)$ & $29 / 45(64.4)$ & 0.856 \\
\hline Stone size $\geq 8 \mathrm{~mm}$ & $61 / 153$ (39.9) & $5 \mathrm{I} / 16 \mathrm{I}(3 \mathrm{I} .7)$ & 0.130 \\
\hline Kidney & $32 / 86(37.2)$ & $31 / 113(27.4)$ & 0.142 \\
\hline Ureter & 29/67 (43.3) & $20 / 48$ (4I.7) & 0.863 \\
\hline
\end{tabular}

Note: ${ }^{\text {TTwo }}$ cases with incomplete data were excluded.

elderly $33.5 \%, P=0.257$ ) and ureteral stones (non-elderly $52.3 \%$ vs elderly $52.7 \%, P=0.964$ ) (Table 2 ). An ROC curve predicted that stones $<0.8 \mathrm{~cm}$ in size had a higher stone-free rate $(P<0.001)$. The Youden's index on the ROC curve with maximal sensitivity $(0.66)$ and specificity $(0.62)$ showed that the threshold stone size for lower stone-free rates was $0.8 \mathrm{~cm}$ (Figure 1). Similar stone-free rates were seen in the elderly and non-elderly regardless of stone size ( $P=0.626$ for stones $<0.8 \mathrm{~cm}$, and $P=0.130$ for stones $\geq 0.8 \mathrm{~cm}$ ) (Table 2). There were no significant differences in the overall retreatment rates (non-elderly $41.6 \%$ vs elderly $37.0 \%, P=0.295$ ) but a higher retreatment rate in kidney stones $<0.8 \mathrm{~cm}$ in size in the non-elderly (non-elderly $30.4 \%$ vs elderly $15.6 \%$, $P=0.046$ ) (Table 3).

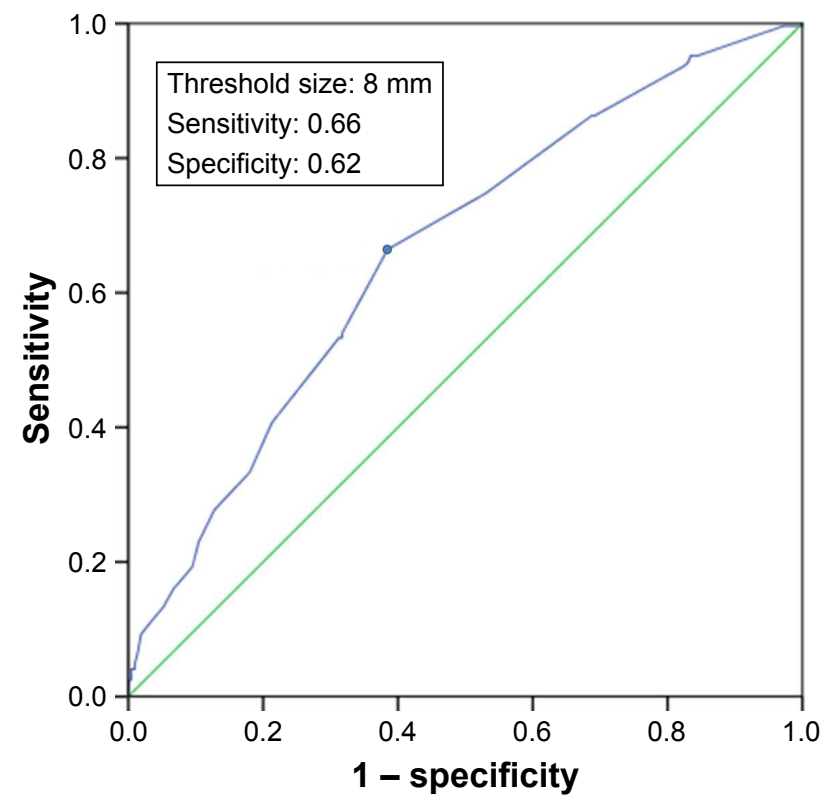

Figure I The ROC curve for stone size to determine which cutoff value predicts the stone-free status after SWL.

Note: Green line, reference line; blue line, stone size in $\mathrm{mm}$

Abbreviations: ROC, receiver operating characteristic; SWL, shock wave lithotripsy.
Table 3 Comparison of retreatment rates by stone site and size

\begin{tabular}{llll}
\hline Variables & $\begin{array}{l}\text { Patients aged } \\
<\mathbf{6 5} \text { years, } \mathbf{n}(\%)\end{array}$ & $\begin{array}{l}\text { Patients aged } \\
\mathbf{2 6 5} \text { years, } \mathbf{n}(\%)\end{array}$ & $\begin{array}{l}\text { P-value } \\
\end{array}$ \\
\hline Overall & $102 / 245(41.6)$ & $88 / 238(37.0)$ & 0.295 \\
Kidney & $5 \mathrm{I} / 119(42.9)$ & $56 / 145(38.6)$ & 0.485 \\
Ureter & $5 \mathrm{I} / 126(40.5)$ & $32 / 93(34.4)$ & 0.360 \\
Stone size $<8 \mathrm{~mm}$ & $28 / 92(30.4)$ & $12 / 77(15.6)$ & 0.024 \\
Kidney & $1 \mathrm{I} / 33(33.3)$ & $4 / 32(12.5)$ & 0.046 \\
Ureter & $17 / 59(28.8)$ & $8 / 45(17.8)$ & 0.192 \\
Stone size $\geq 8 \mathrm{~mm}$ & $74 / 153(48.4)$ & $76 / 161(47.2)$ & 0.837 \\
Kidney & $40 / 86(46.5)$ & $52 / 113(46.0)$ & 0.945 \\
Ureter & $34 / 67(50.7)$ & $24 / 48(50)$ & 0.937 \\
\hline
\end{tabular}

Thirty-eight (15.5\%) non-elderly patients and $56(23.5 \%)$ elderly patients had complications $(P=0.026)$. Elderly patients with ureteral stones, especially those $\geq 0.8 \mathrm{~cm}$ in size, had a higher complication rate than non-elderly patients (non-elderly $3.0 \%$ vs elderly $31.2 \%, P<0.001$ ) (Table 4 ). Logistic regression analysis showed that when compared with the non-elderly, elderly patients with ureteral stones of any size (odds ratio [OR] $=6.57,95 \%$ confidence interval [CI]: $2.55-16.92$ ) and ureteral stones $\geq 0.8 \mathrm{~cm}$ in size (OR $=14.77,95 \%$ CI: 3.19-68.48) had significantly increased risks of complications (Table 5). An ROC curve predicted that patients aged $>65$ years had a higher complication rate $(P<0.005)$. The Youden's index on the ROC curve with maximal sensitivity $(0.60)$ and specificity $(0.53)$ showed that the threshold age for higher complication rates was 65 years (Figure 2). This threshold age coincided with the preset age limit of 65 years, confirming that elderly patients had higher complication rates. The most common complication was post-SWL-related flank pain (grade I), which occurred in $38(15.5 \%)$ non-elderly patients and $52(21.8 \%)$ elderly patients $(P=0.06)$. Post-SWL-related pain was controlled with intravenous or intramuscular nonsteroidal anti-inflammatory drugs in all cases. In the

Table 4 Comparison of complication rates by stone site and size

\begin{tabular}{|c|c|c|c|}
\hline Variables & $\begin{array}{l}\text { Patients aged } \\
<65 \text { years, } \mathrm{n}(\%)\end{array}$ & $\begin{array}{l}\text { Patients aged } \\
\geq 65 \text { years, } n \text { (\%) }\end{array}$ & $P$-value \\
\hline Overall & $38 / 245(15.5)$ & $56 / 238(23.5)$ & 0.026 \\
\hline Kidney & $32 / 119(26.9)$ & $33 / 145$ (22.8) & 0.438 \\
\hline Ureter & $6 / 126(4.8)$ & $23 / 93(24.7)$ & $<0.001$ \\
\hline Stone size $<8 \mathrm{~mm}$ & $15 / 92(16.3)$ & I4/77 (I8.1) & 0.747 \\
\hline Kidney & $11 / 33(33.3)$ & $6 / 32(18.8)$ & 0.181 \\
\hline Ureter & $4 / 59(6.8)$ & $8 / 45(17.8)$ & 0.082 \\
\hline Stone size $\geq 8 \mathrm{~mm}$ & $23 / 153(15.0)$ & $42 / 16 I(26.0)$ & 0.016 \\
\hline Kidney & $21 / 86(24.4)$ & $27 / 113(23.9)$ & 0.932 \\
\hline Ureter & $2 / 67(3.0)$ & $\mid 5 / 48(3 \mid .2)$ & $<0.001$ \\
\hline
\end{tabular}


Table 5 Risk of complications in patients aged $\geq 65$ years compared with patients aged $<65$ years

\begin{tabular}{cll}
\hline & OR (95\% Cl) & P-value \\
\hline Overall & $1.68(1.06-2.65)$ & 0.027 \\
Kidney & $0.80(0.46-1.40)$ & 0.438 \\
Ureter & $6.57(2.55-16.92)$ & $<0.00 \mathrm{I}$ \\
Stone size $<8 \mathrm{~mm}$ & $1.14(0.5 \mathrm{I}-2.54)$ & 0.747 \\
Kidney & $0.46(0.15-1.45)$ & 0.186 \\
Ureter & $2.97(0.83-10.59)$ & 0.093 \\
Stone size $\geq 8 \mathrm{~mm}$ & $1.99(1.13-3.5 \mathrm{I})$ & 0.017 \\
Kidney & $0.97(0.50-1.87)$ & 0.932 \\
Ureter & $14.77(3.19-68.48)$ & $0.00 \mathrm{I}$ \\
\hline
\end{tabular}

Abbreviations: $\mathrm{Cl}$, confidence interval; $\mathrm{OR}$, odds ratio.

elderly group, one patient had a urinary tract infection and one patient had nausea postoperatively. Two elderly patients $(0.8 \%)$ had subcapsular renal hematomas. One patient received blood transfusion (grade II), and the other patient underwent trans-arterial embolization at the intensive care unit (grade IV).

\section{Discussion}

This study showed that SWL for upper urinary tract stones was equally efficacious in elderly and non-elderly patients, but a higher complication rate was seen in the elderly. Previous studies yielded conflicting results. $\mathrm{Ng}$ et al showed that in the elderly, the stone-free rate was significantly lower for renal stones but not for ureter stones. ${ }^{4}$ Dhar et al showed

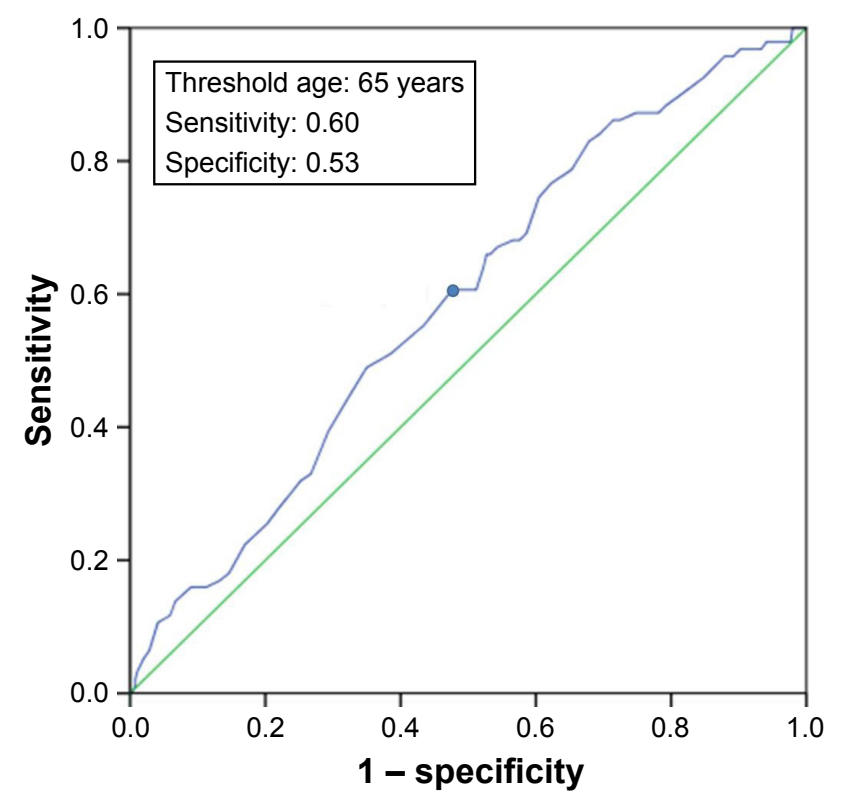

Figure 2 The ROC curve for patient age to determine which cutoff value predicts the complication after SWL.

Note: Green line, reference line; blue line, patient age in years.

Abbreviations: ROC, receiver operating characteristic; SWL, shock wave lithotripsy. that the risk of complications after SWL was higher in the elderly population. ${ }^{5}$

This study is important because the elderly is a unique population, and medical practitioners must decide which treatment modalities are efficacious and safe in this group of patients with higher inherent surgical risks. Ackermann et al showed that patients aged $>60$ years had the lowest stone-free rate for renal stones compared with all other age groups. ${ }^{6}$ Abdel-Khalek et $\mathrm{al}^{7}$ revealed that patients aged $>40$ years had a significantly lower stone-free rate for renal stones than patients aged $<40$ years and also showed that patients' age was not a significant factor for ureter stones. A study by Kimura and Sasagawa ${ }^{8}$ showed that younger age and small stone size were prognostic factors determining better stone clearance. However, another study by Halachmi and Meretyk also showed that patients' age was not a predicting factor for ureteral stone clearance. ${ }^{9}$ AbdelKhalek et al reported that renal and ureteral stones $\leq 1 \mathrm{~cm}$ in size were associated with a higher stone-free rate compared with those $>1 \mathrm{~cm}$ in size. This study also showed that stone size was a significant factor in stone clearance rates. In this study, the non-elderly group had a higher retreatment rate for stones $<0.8 \mathrm{~cm}$ and renal stones $<0.8 \mathrm{~cm}$ in size $(P<0.05)$. However, these different retreatment rates between the two groups could be due to bias, as decision for further surgical intervention was based on patients' clinical symptoms, patients' willingness to undergo further treatment, and various urologists' clinical experience and practice.

For the comparison of treatment outcomes between the two groups, stratification of the results according to stone size and stone site was recommended by Clayman et al. ${ }^{10}$ In the present study, stratification analysis showed that the elderly and non-elderly groups had comparable outcomes when comparisons were made according to different stone sizes and stone sites.

SWL may potentially induce renal trauma. ${ }^{11}$ Age, obesities, coagulopathies, thrombocytopenia, diabetes mellitus, coronary heart disease, and preexisting hypertension are the risk factors for SWL-related renal trauma. In this study, two $(0.8 \%)$ patients in the elderly group developed renal hematomas. One was an 82-year-old male with a $5 \mathrm{~cm}$ renal stone, who was hardly indicated for SWL. He was given a blood transfusion. The other was a 70-year-old female with a $1 \mathrm{~cm}$ renal stone, who had coronary artery disease, diabetes mellitus, and hypertension. She underwent trans-arterial embolization and was admitted to the intensive care unit. Both the patients recovered uneventfully. Dhar et $\mathrm{al}^{5}$ reported the incidence of renal hematoma after electromagnetic 
lithotripsy for renal stones to be $4.1 \%$, and the probability of hematoma increased 2.2 times for every 10-year increase in patient's age. Sheir et $\mathrm{a}^{12}$ reported an incidence of renal hematoma of $0.5 \%(4 / 694)$. Due to functional and structural injury to the kidney, SWL of kidney stones is more likely to cause significant complications.

A large national SWL database in New Zealand showed that common post-SWL complications included pain managed with intravenous or suppository analgesics $(5.9 \%)$, perinephric hematoma $(0.2 \%)$, urinary tract infection $(1.1 \%)$, urosepsis $(0.04 \%)$, and hospital admission $(6.8 \%) .{ }^{13}$ In the present study, the elderly group had a higher complication rate than the non-elderly group $(23.8 \%$ vs $15.5 \%, P<0.05)$. Jamshaid et $\mathrm{al}^{11}$ reported an incidence of renal colic pain of $3.6 \%(10 / 274)$, whereas Sheir et al ${ }^{12}$ reported an incidence of renal colic pain of $9.6 \%$. Renal colic pain was the most common complication, and it occurred in $18.6 \%$ of the patients included in this study. They received intravenous or intramuscular analgesics and recovered well.

A significantly higher complication rate was seen in elderly patients with ureteral stones $\geq 0.8 \mathrm{~cm}$ in size. This may be due to the larger ureteral stones being disintegrated into more pieces and the higher probability of pain caused by these fragments passing through the ureter. In the analysis, it was found that elderly patients with ureteral stones, especially those $>0.8 \mathrm{~cm}$, had a higher risk of complications (mostly pain) than non-elderly patients. Sokolis et $\mathrm{al}^{14}$ showed that aging increased the thickness of the ureter muscular wall relative to the mucosal layers. This thickened ureter with a possibly narrower lumen could explain why elderly patients suffered from more pain when passing stones compared with non-elderly patients.

This study showed that patients aged $\geq 65$ years had a higher complication rate $(P<0.005)$. Compared with other studies of patients of all ages, the present study in elderly patients showed a higher complication rate after SWL.

Comparative studies are difficult as most of them are retrospective and may contain many potential biases. Studies may be multicentered or single centered. In addition, SWL protocols (shock wave machines, energy, rate, frequency, and operative time), preoperative protocols, anesthesia protocols, follow-up protocols (time for post-SWL plain film time or kidney ultrasound), and retreatment protocols (time for secondary intervention) may differ from institution to institution. Moreover, experience of the operating staffs may be different. These factors gathering together may affect the final outcomes. ${ }^{15,16}$ Single-center studies are more likely to provide a conclusive result than multicenter studies due to relatively minimized bias. ${ }^{17}$ Our study was a retrospective single-center study. All SWL treatments were performed by the same two experienced operators operating both machines in two branches of our hospital, and all the patients' medical records were reviewed by a single researcher. The consistency of these data was high, and potential biases were minimized as much as possible. The limitations of this study were 1) the retrospective nature of the study, 2) the small number of patients, 3) the absence of guidelines for stent placement before SWL, 4) the lack of computed tomography exams from which the hardness of the stones could be calculated, and 5) the lack of information about stone composition.

\section{Conclusion}

This study showed that SWL of upper urinary tract stones in elderly patients had comparable stone-free rates and retreatment rates to non-elderly patients, but complication rates were higher in the elderly. However, larger randomized studies are needed to verify these results. Nevertheless, results from the present study may serve as reference in the management of upper urinary tract stones in the ever-growing number of elderly patients.

\section{Disclosure}

The authors report no conflicts of interest in this work.

\section{References}

1. Turk C, Petrik A, Sarica K, et al. EAU Guidelines on Interventional Treatment for Urolithiasis. Eur Urol. 2016;69(3):475-482.

2. Wan He DG, Paul Kowal. An Aging World: 2015. Government Publishing Office, Washington, DC, International Population Reports, P95/16-1; 2016. Available from: https://www.census.gov/content/ dam/Census/library/publications/2016/demo/p95-16-1.pdf. Accessed October 12, 2016

3. Romero V, Akpinar H, Assimos DG. Kidney stones: a global picture of prevalence, incidence, and associated risk factors. Rev Urol. 2010 12(2-3):e86-e96.

4. Ng CF, Wong A, Tolley D. Is extracorporeal shock wave lithotripsy the preferred treatment option for elderly patients with urinary stone? A multivariate analysis of the effect of patient age on treatment outcome. BJU Int. 2007;100(2):392-395.

5. Dhar NB, Thornton J, Karafa MT, Streem SB. A multivariate analysis of risk factors associated with subcapsular hematoma formation following electromagnetic shock wave lithotripsy. $J$ Urol. 2004;172(6 Pt 1): 2271-2274.

6. Ackermann DK, Fuhrimann R, Pfluger D, Studer UE, Zingg EJ. Prognosis after extracorporeal shock wave lithotripsy of radiopaque renal calculi: a multivariate analysis. Eur Urol. 1994;25(2): 105-109.

7. Abdel-Khalek M, Sheir KZ, Mokhtar AA, Eraky I, Kenawy M, Bazeed M. Prediction of success rate after extracorporeal shock-wave lithotripsy of renal stones - a multivariate analysis model. Scand J Urol Nephrol. 2004;38(2):161-167. 
8. Kimura M, Sasagawa T. [Significance of age on prognosis in patients treated by extracorporeal shock wave lithotripsy]. Nihon Hinyokika Gakkai zasshi.Jpn J Urol. 2008;99(4):571-577. Japanese [with English abstract].

9. Halachmi S, Meretyk S. Shock wave lithotripsy for ureteral stones in elderly male patients. Aging Male. 2006;9(3):171-174.

10. Clayman RV, McClennan BL, Garvin TJ, Denstedt JD, Andriole GL. Lithostar: an electromagnetic, acoustic shock wave unit for extracorporeal lithotripsy. In: Lingeman JE, Newman DM, editors. Shock Wave Lithotripsy 2: Urinary and Biliary Lithotripsy. Boston, MA: Springer US; 1989:403-409.

11. Jamshaid A, Ather MH, Hussain G, Khawaja KB. Single center, single operator comparative study of the effectiveness of electrohydraulic and electromagnetic lithotripters in the management of 10- to 20-mm single upper urinary tract calculi. Urology. 2008;72(5):991-995.

12. Sheir KZ, Madbouly K, Elsobky E. Prospective randomized comparative study of the effectiveness and safety of electrohydraulic and electromagnetic extracorporeal shock wave lithotriptors. J Urol. 2003; 170(2 Pt 1):389-392.
13. Alexander CE, Gowland S, Cadwallader J, Reynard JM, Turney BW. Shock wave lithotripsy (SWL): outcomes from a national SWL database in New Zealand. BJU Int. 2016;117(Suppl 4):76-81.

14. Sokolis DP, Petsepe DC, Papadodima SA, Kourkoulis SK. Age- and region-related changes in the biomechanical properties and composition of the human ureter. J Biomech. 2017;51:57-64.

15. Tolley DA. Consensus of lithotriptor terminology. World J Urol. 1993; 11(1):37-42.

16. Fialkov JM, Hedican SP, Fallon B. Reassessing the efficacy of the Dornier MFL-5000 lithotriptor. J Urol. 2000;164(3 Pt 1):640-643.

17. Ng CF, Thompson TJ, McLornan L, Tolley DA. Single-center experience using three shockwave lithotripters with different generator designs in management of urinary calculi. J Endourol. 2006;20(1):1-8.
Clinical Interventions in Aging

\section{Publish your work in this journal}

Clinical Interventions in Aging is an international, peer-reviewed journal focusing on evidence-based reports on the value or lack thereof of treatments intended to prevent or delay the onset of maladaptive correlates of aging in human beings. This journal is indexed on PubMed Central, MedLine,

\section{Dovepress}

CAS, Scopus and the Elsevier Bibliographic databases. The manuscript management system is completely online and includes a very quick and fair peer-review system, which is all easy to use. Visit http://www.dovepress. com/testimonials.php to read real quotes from published authors. 